\title{
The efficacy of podiatric orthoses as an adjunct to the treatment of plantar ulceration in leprosy
}

\author{
H. CROSS, ${ }^{*}$ S. SANE, A. DEY $\dagger \&$ \\ V. N. KULKARNI† \\ *Queen Margaret College, Edinburgh EH6 8HF; and $\dagger$ Dr Bandor- \\ awalla Leprosy Hospital, Pune, India
}

Accepted for publication 2 December 1994

\begin{abstract}
Summary This study examines the outcome of a mangement approach to plantar ulceration secondary to leprotic neuropathy. Locally-available resources were used to produce podiatric orthoses which were supplied to an experimental group. The effects on healing time and quality of healing were compared with a control group. Both groups were ambulant (the programme sought not to interfere with socioeconomic independence). Using standard nonparametric methods of analysis, it was demonstrated that the effects on healing rate, attributed to the experimental intervention, were highly significant. Over a 7 -month period, $57 \%$ of the experimental group ulcers healed, while only $12.5 \%$ of the control group ulcers healed. The experimental intervention also demonstrated a positive effect on the quality of ulcer healing. This effect was not reflected in the control group.
\end{abstract}

\section{Introduction}

Disability prevention, as an issue, is gaining status as awareness of the potential for leprosy eradication as a health care problem becomes increasingly plausible. This study developed as a need was perceived to improve the current approach to the treatment of simple ulcers whilst observing the resource limitations of an institution in a developing country was recognized.

The therapeutic rationale supporting the intervention of mechanical therapy for the neuropathic foot was based on findings reported in the literature. Other investigators have sought to explain the action of orthotic devices, or footwear modifications, by examining the effects of intervention on the distribution of pressure or on the redirection of the centre of force. ${ }^{1-11}$ These investigators have validated principles of deflection, cushioning, increased weight-bearing area or alteration of the rearfoot alignment with the supporting surface.

Recognizing the significance of mechanical factors as agents of destruction threatening the neuropathic foot created a challenge that has been vigorously pursued 
since the late $1950 \mathrm{~s}^{1,12-15}$ The concern to develop conservative treatment of plantar ulceration arose from the recognition that ulceration was an initiating factor precipitating the apparent vortex of destruction, all too commonly culminating in amputation. ${ }^{16}$

Extensive groundwork has been conducted to demonstrate desirable design features, and approaches to treatment that would address pathomechanical characteristics of ulceration. Plaster of Paris casting and rest have demonstrated efficacy. ${ }^{17-18}$ However, the effectiveness of the approach is less satisfactory as recurrence is common, and repeated or prolonged treatment has undesirable social and medical consequences.

Many of the early footwear innovations were laudable. It is broadly agreed that a rigid-soled shoe with a carefully placed rocker effectively reduces focal pressure (generally accepted to be an aetiological factor effecting ulceration). The beneficial effects are explained by the principle that a rigid-soled shoe pivoting on a carefully placed rockerbar will cause loading to be redistributed over a larger area of the foot. ${ }^{1} \mathrm{~A}$ consequence is that the loading time for greater areas of the foot in contact with the rigid shoe will be increased thereby decreasing focal concentrations of force. This assumption is not necessarily correct as the effect of mixed variables requires consideration, e.g. the functional adaptations of the structure of the foot as a kinematic response effected by peculiarities of gait.

Brand ${ }^{19}$ has expressed reserve in his opinion of orthotic intervention for neuropathic feet. His concern was that improperly placed devices may increase focal pressures and exacerbate ulceration. Whilst recognizing this hazard, the results of this study illustrate that orthotic intervention can significantly reduce the moribidity of ulceration. Although regression of ulceration in a minority of cases may be attributed to damage by devices it would appear that a significant majority benefited by the intervention. It is accepted that the significance of the findings demonstrated in this investigation may be an indication of professional expertise and may therefore be limited in their application among the wider population. The training of leprosy workers and the assessment of their ability to prescribe and manufacture devices may demonstrate the wider potential benefit of this form of intervention.

\section{Method}

SAMPLE SELECTION

A sample of 71 subjects was drawn from outpatients and employees at the Dr Bandorawalla Leprosy Hospital, Kondhawa, Pune, India. All subjects presented with sequelae of leprosy including, anaesthesia and plantar ulceration. All were suitable for prescription of an individual orthosis from a wide range of podiatric orthoses.

The criteria for subject inclusion excluded bedrest patients, patients being treated with plastercasting, patients presenting with acute osteomyelitis or patients for whom specialized orthopaedic footwear had been prescribed. The selection criteria for both experimental and control groups were identical. There was no selection bias. The supply of orthoses depended on the mutual availability of subjects, the interpreter and the researcher.

We fitted 37 subjects with orthoses and allocated them to the experimental group; 34 were not offered orthoses and these were allocated to the control group. By March, 
5 subjects had dropped out of the experimental group and a further subject was excluded due to intervention with plastercast treatment. These exclusions reduced the number of subjects to 31 , reducing the number of ulcerated feet for analysis to 35 and the number of ulcers to 40 (4 feet presented with multiple ulcers). In the control group, 3 subjects had dropped out, reducing the number of subjects to 31 , the number of ulcerated feet for analysis to 35 and the number of ulcers to 39 (4 feet presented with multiple ulcers).

\section{METHODS OF ASSESSMENT AND EVALUATION}

All subjects were assessed for sensory loss and deformity during the initial visit. For this study the patients' response to pain and vibration was tested using the techniques described by Bickerstaff \& Spillane. ${ }^{20}$ A $128 \mathrm{~Hz}$ tuning fork was used to test vibration and the pinprick test was applied to test for pain response. Findings relating to sensory loss and observations of deformity were recorded diagrammatically on foot maps as described by Watson. ${ }^{21}$

The assessment of ulceration followed standardized criteria established by Wall ${ }^{22}$ who described general features of ulceration that indicated stages of degeneration, chronicity or resolution.

After recording the number of lesions, the size of individual ulcers was measured. Measurements were recorded at increments of $0.25 \mathrm{~cm}$. Size was recorded as:

The greatest distance between 2 points on the edge, longitudinally.

The greatest distance between 2 points on the edge, horizontally.

The area of ulceration was then calculated using the formula:

$$
(\text { Horizontal length }+ \text { Longitudinal length }) \times 0 \cdot 785^{23,24}
$$

Ulceration was further described by categorical rating of edge and surface morphology.

\section{SUPPLY OF ORTHOSES}

The devices issued incorporated features aimed at addressing both palliative and functional demands (Figure 1). Prescription was based on observations of gait and examination of foot structure. The choice of materials used was determined by local supply. Devices were fabricated from microcellular rubber to accommodate the requirements of individual subjects. The supply of elasticated removable devices was limited to subjects demonstrating an uncompromised vascular supply and sufficient manual dexterity. All other devices were adhered to subject's footwear.

The subject's choice of footwear was respected, however, and if damaged it was either replaced or repaired. All changes to, or of, footwear over the experimental period were recorded. The use of gauze swabs secured by tape or minimal bandage was domonstrated to experimental group subjects. Where ulcers did not present with excessive exudate experimental group subjects were encouraged to dress ulcers with zinc tape. This approach has been shown to demonstrate an effect not significantly different from gauze bandage. ${ }^{25}$ Control group subjects continued to dress ulcers with gauze bandage. 
Filler Pad Incorporating Valgus Dome And Cut Away For Pressure Deflection

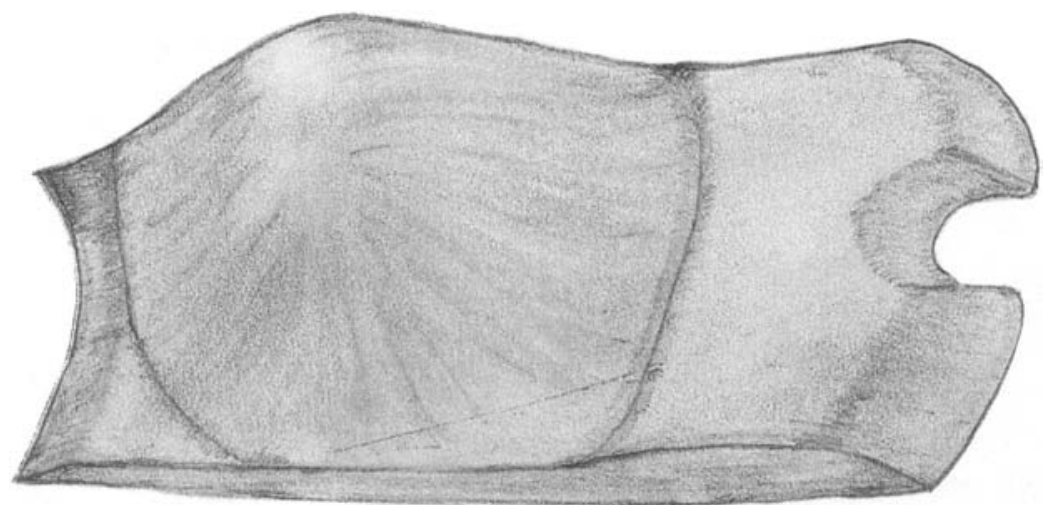

Filler Pad Incorporating Valgus Dome With Forefoot Modification And Cut Away For Pressure Deflection

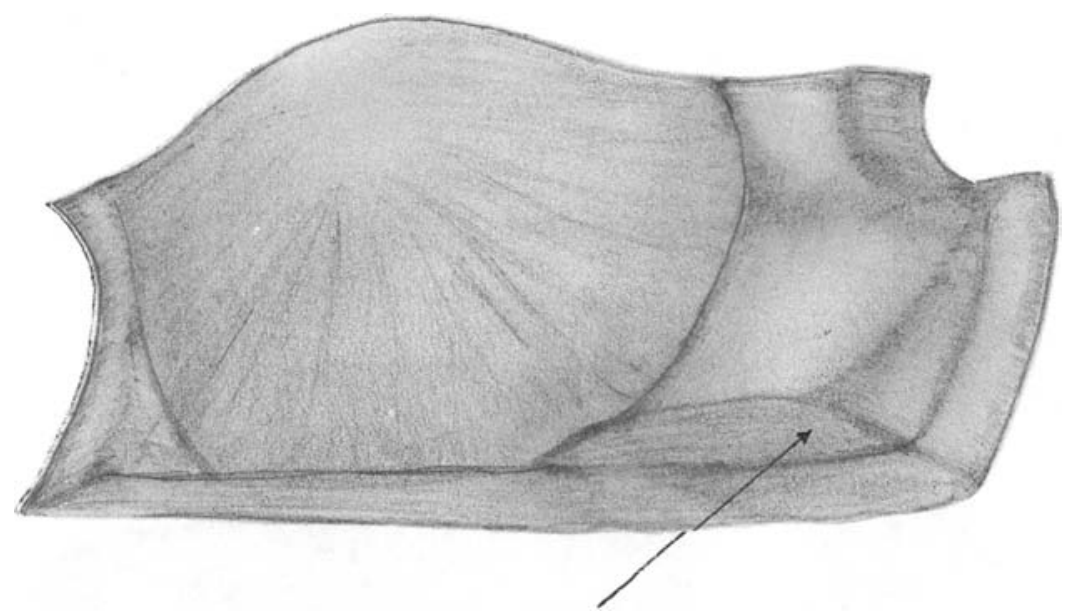

Forefoot Wedge To Accomodate Forefoot Valgus Deformity

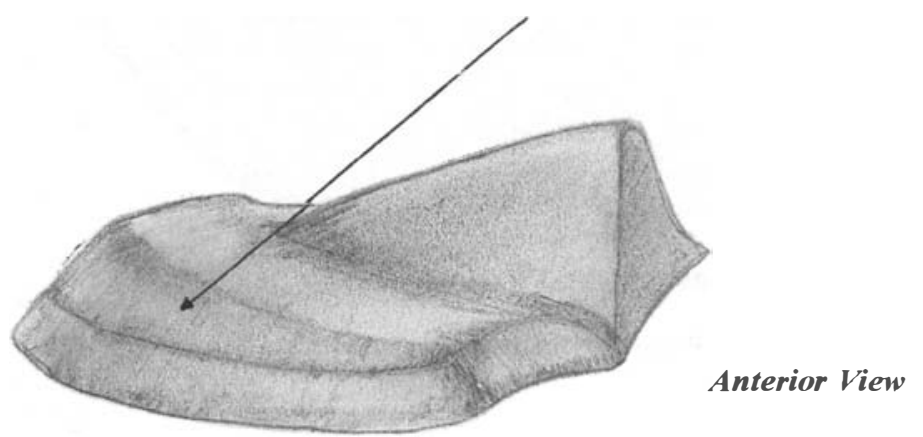

Figure 1. Orthoses supplied to the experimental group, July-August 1994. 


\section{Plantar Metatarsal Pad}

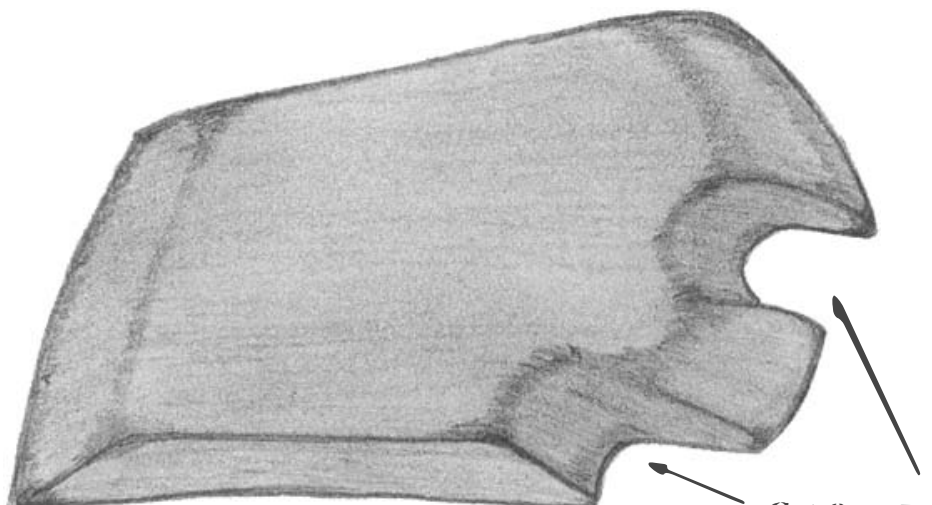

Cut Outs For Pressure Deflection At The Third And Fifth Metatarsal Heads

\section{Removable Plantar Metatarsal Pad}

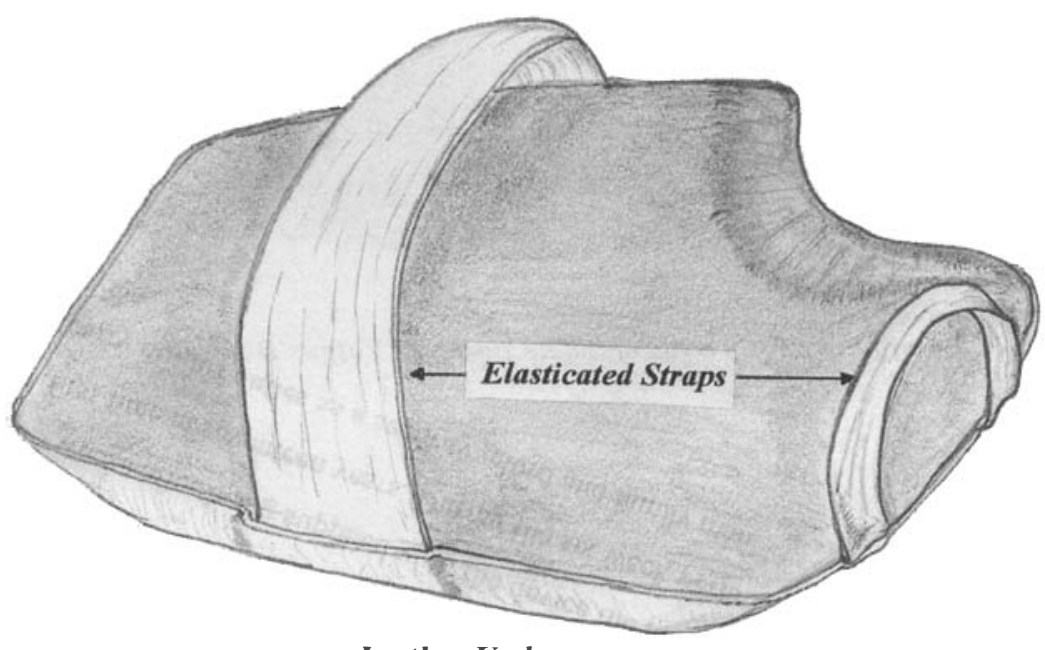

Leather Undercover

Figure 1. (Continued). 
Filler Pad Incorporating Valgus Dome And Anterior Rocker Bar

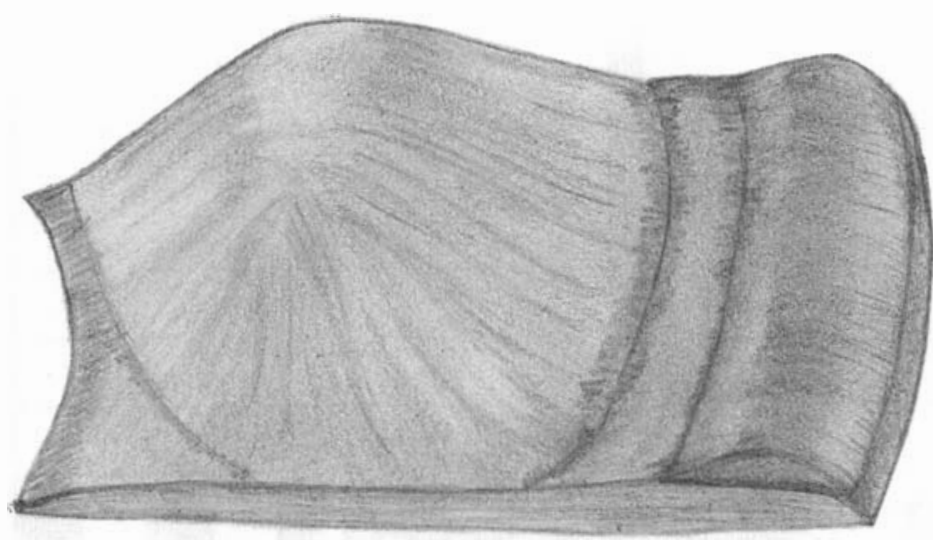

Filler Pad Incorporating $\boldsymbol{A}$ Window

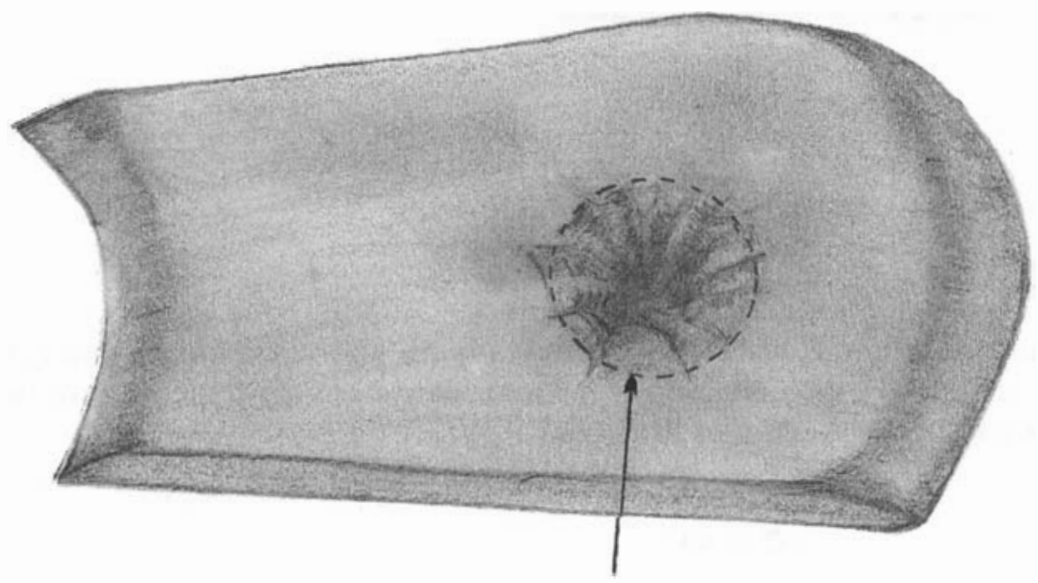

Cavity Excavated Beneath Device

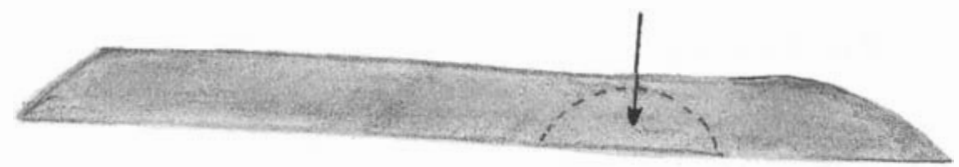

Lateral View

Figure 1. (Continued). 


\section{Distribution of Ulcers : JulfAug 93}

Categorised for Presentation

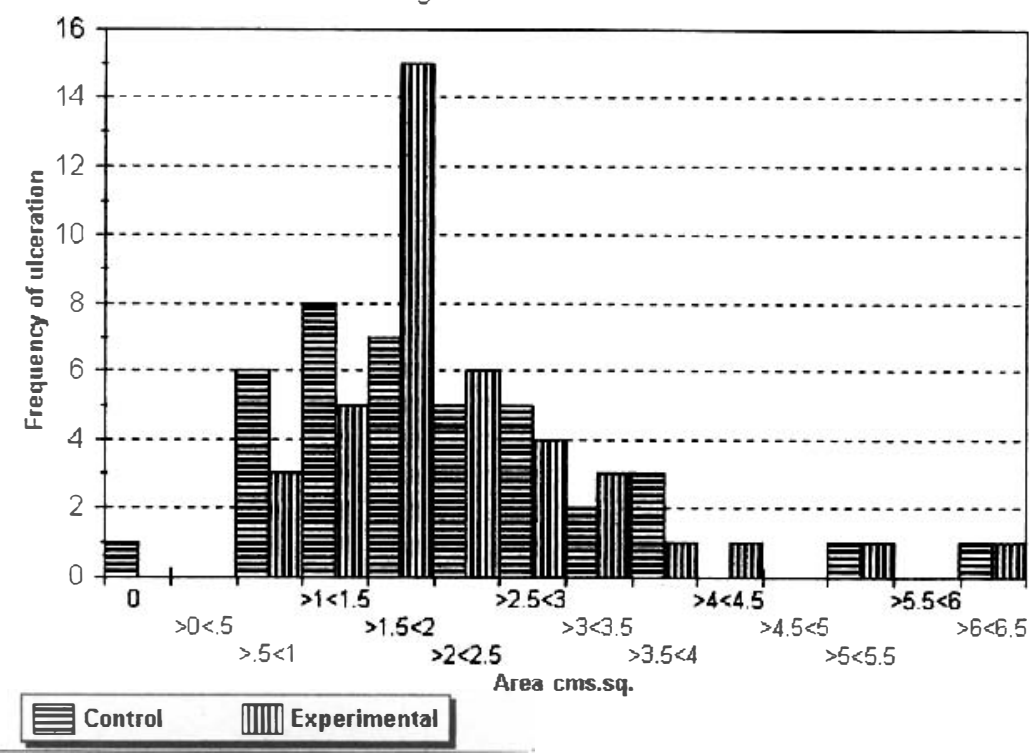

Figure 2.

\section{BASELINE DATA}

\section{Ulcer size}

The Mann-Whitney $U$-test was used to compare ulceration dimensions (expressed as area of ulceration) between experimental and control groups at the onset of the trial. The data represented here excludes drop outs:

$$
\begin{aligned}
n 1 & =39, \\
n 2 & =40, \\
\mathrm{U} & =758, \\
\text { 'U' } & =782 \cdot 5,95 \% \mathrm{CI}=-0 \cdot 39 \text { to } 0 \cdot 39, \mathrm{NS} .
\end{aligned}
$$

(See Figure 2 and Tables 1 and 2.)

Table 1. Comparison of edge morphology

\begin{tabular}{lcclc}
\hline Type & Undermined & Indurated & Shelved & Total \\
\hline Experimental & 20 & 15 & 5 & 40 \\
Control & 25 & 11 & 3 & 39 \\
Total & 45 & 26 & 8 & 79 \\
$\chi^{2}$ & $0 \cdot 43 / \mathrm{NS}$ & $0 \cdot 39 / \mathrm{NS}$ & $0 \cdot 36 / \mathrm{NS}$ & \\
\hline
\end{tabular}


Table 2. Comparison of surface morphology

\begin{tabular}{lcccc}
\hline Type & Slough & Granulation & Perforating & Total \\
\hline Experimental & 9 & 20 & 11 & 40 \\
Control & 13 & 21 & 4 & 39 \\
Total & 22 & 41 & 15 & 79 \\
$\chi^{2}$ & $0 \cdot 83 / \mathrm{NS}$ & $0 \cdot 06 / \mathrm{NS}$ & $0 \cdot 2 \cdot 8 / p=0 \cdot 08$ & \\
\hline
\end{tabular}

\section{Morphology of ulceration}

The Fisher-Irwin exact test was used to compare the edges and surfaces between both groups. The findings demonstrate that there was no significant difference between groups.

\section{Results}

\section{ULCER SIZE}

Data relating to the March 1994 assessment of the control group were compared with those of the experimental group during the same period.

The experimental group data displayed that of 40 ulcers $23(57.5 \%)$ had healed. The

\section{Distribution of Ulcers: March 94}

Categorised for Presentation

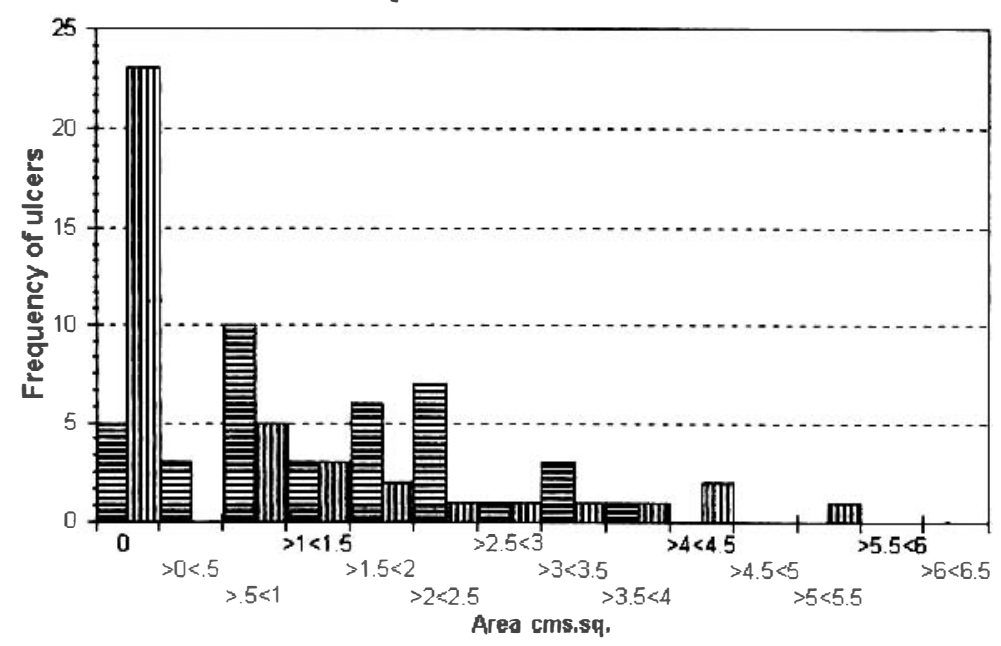

Control IIII) Experimental

Figure 3. 
control group data displayed that of 39 ulcers $5(12.5 \%)$ had healed. Comparison of data representing the ulcer area in March 1994

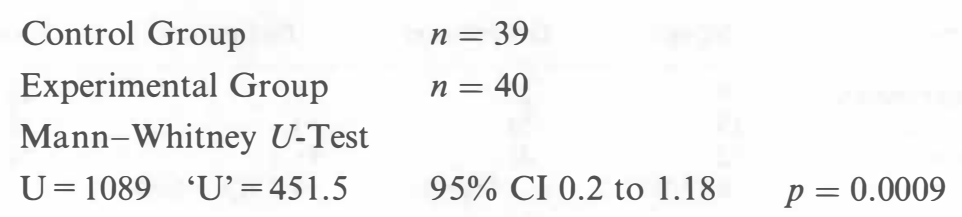

(See Figure 3.)

UNRESOLVED ULCERS

Comparison of data representing the area of unresolved ulcers in March 1994.

Control Group $n=34$;

Experimental Group $n=16$.

Mann-Whitney $U$-test

$\mathrm{U}=337 \cdot 5$, 'U' $=96,95 \% \mathrm{CI}=0.02$ to $1 \cdot 18$, NS.

\section{Analysis}

The results demonstrate that a highly significant reduction in the mean area of ulceration was demonstrated between both groups in March. This reduction is explained by the 5 -fold number of healed ulcers in the experimental group when compared with the control group. However, the unhealed ulcers in the experimental group were not significantly different in size from the unhealed ulcers in the control group.

\section{CHARACTERISTICS OF ULCERATION}

A hypothesis suggesting that positive changes in ulcer morphology were concurrent with changes in the area of ulceration was tested. $\chi^{2}$ was used to demonstrate whether the trend in mean scores between the area of ulceration at each assessment and groups categorized as regressive, static and progressive were significantly different.

Observations relating to undermined edges were grouped with observations relating to perforating and hypergranulating surfaces and were categorized as regressive.

Observations relating to indurated edges were grouped with observations relating to sloughing ulcers and were categorized as static.

Observations relating to shelved edges were grouped with observations relating to granulation and were categorized as progressive. (See Tables 3 and 4.)

\section{EXPERIMENTAL GROUP}

Area and progressive:

$$
\chi^{2} \text { for trend in mean scores }=5 \cdot 91, \mathrm{df} 1, p=0 \cdot 01 .
$$

Area and static

$$
\chi^{2} \text { for trend in mean scores }=0 \cdot 28, \mathrm{df} 1, \mathrm{NS} .
$$


Area and regressive

$$
\chi^{2} \text { for trend in mean scores }=2 \cdot 35 \text {, df } 1, \mathrm{NS} .
$$

Table 3. Experimental group: changes in ulcer characteristics relative to area*

\begin{tabular}{lcccc}
\hline Group & July & September & December & March \\
\hline Regressive & 32 & 7 & 12 & 6 \\
$\begin{array}{l}\text { Static } \\
\text { Progressive }\end{array}$ & 35 & 16 & 18 & 10 \\
$\begin{array}{l}\text { Total area of all } \\
\text { observations }\end{array}$ & 13 & 37 & 16 & 18 \\
& $83 \cdot 76$ & $51 \cdot 52$ & $33 \cdot 72$ & $37 \cdot 07$ \\
\hline
\end{tabular}

\footnotetext{
* The increase in area from December to March (experimental group) was not significant. $n 1=23, n 2=17 \quad$ Mann-Whitney $U$-test, $U=253 \cdot 5$, 'U' $=86 \cdot 5,95 \% \mathrm{CI}=0$ to $1 \cdot 56, \mathrm{NS}$.
}

Table 4. Control group: changes in ulcer characteristics relative to area

\begin{tabular}{lcccc}
\hline Group & July & September & December & March \\
\hline Regressive & 28 & 19 & 17 & 18 \\
Static & 24 & 24 & 28 & 25 \\
Progressive & 24 & 23 & 23 & 25 \\
$\begin{array}{l}\text { Total area of all } \\
\text { observations }\end{array}$ & $80 \cdot 12$ & $68 \cdot 73$ & 63.04 & $54 \cdot 4$ \\
& & & & \\
\hline
\end{tabular}

\section{Analysis}

As the experimental group area of ulceration decreased at consecutive assessments, the increase in the number of observations relating to progressive characteristics was significant. Conversely the comparative trends in mean scores relating to area and regressive or static characteristics were not significant. This finding suggests that these characteristics decreased as the area of ulceration decreased.

Difference in trend of mean scores relating to positive characteristics presented by the control group were not significant.

These results suggest that the intervention may have imposed a stabilizing effect on ulcers in the experimental group creating conditions in which the healing process was enhanced.

\section{Confounding Variables}

Care was taken to examine the potential effects of other independent variables to confound the results. The effects of deformity, sites of ulcertion and occupation were examined and found not to have demonstrated a significant effect on the results. 
Different types of orthotic device were examined and found not to have favoured sub groups within the Experimental group.

\section{Discussion}

The results presented indicate that after a 6-month period subjects in the experimental group were more likely to present with healed ulcers $(52.5 \%$ healed) than subjects in the control group ( $12 \cdot 5 \%$ healed).

Favourable alterations in the morphology of ulceration, concurrent with a reduction in area was a feature of experimental group ulcers. The trend was not reflected in the control group where a reduction in ulcer area was not accompanied by changes characteristic of a sound healing process. On this basis, a statement suggesting that orthotic intervention enhances the quality of healing is supported. The major implications of this finding are 2-fold. Primarily, orthotic treatment reduces the time required to heal an ulcer (when compared with gauze dressing treatment). Furthermore, by attenuating or reducing the effects of irritation during healing the opposition to the homeostatic response to ulceration is reduced.

\section{SCAR TISSUE}

During the maturation or remodelling phase of tissue repair there is a decline in concentration of fibroblasts. A complex reorientation activity organizes random collagen fibres into a system of optimally oriented fibres. The eventual result is the consolidation of scar tissue, which displays a maximum strength of $20 \%$ less than that exhibited by intact skin. ${ }^{26,27}$

The rapid process of synthesis and lysis of collagen in the early stages of healing is reversed during maturation such that the rate of lysis exceeds that of synthesis. The nature of biochemical bonding is also alerted. This results in the establishment of stronger molecular structures and links between collagen fibrils and ground substance. It is fundamental therefore to promote rapid healing to discourage the excessive linkage of collagen and ground substance. The weaker bonding of collagen in developing scar allows for limited stretch as a response to stress. With maturation scar tissue does become less resilient. However, recurrent trauma during the maturation phase leads to increasing deposits of more dense and toughened mature scar organized into progressively less resilient tissue. Brand ${ }^{19}$ has discussed the problem of scarring as a response to recurrent trauma. He has related the common recurrence of ulceration to the cumulative degeneration of skin into scar tissue.

The application of plastercasts have proven efficacious in the rapid healing of neuropathic ulceration. ${ }^{16}$ Anecdotal evidence suggests that ulcers may heal within a period of 3 weeks. Birke et al. ${ }^{17}$ recorded that patients treated with walking casts presented with ulcers that healed in 37.7 days. However, the recurrence of ulceration following this procedure is common, particularly where predisposing mechanical factors have not been addressed subsequent to treatment. A more subtle problem may be the effect of immobilization on the healing response.

The realignment of collagen fibres is thought to be a response to pressure. When pressure is applied collagen releases 'piezoelectric substances. ${ }^{26}$ It is postulated that 
these stress generated voltages are responsible for the realignment and general maintenance of collagen. This provides an important perspective to wound healing as it suggests that the strength of healed skin is determined by biomechanical stresses acting on healing time. The fragility of healing resulting from immobility may be reduced by permitting careful movement of healing tissue. It is not suggested here that orthotic intervention of the type under review is particularly subtle. However, it may be that by permitting the subject to be fully ambulant whilst controlling traumatic mechanical factors, the maturation phase of the healing process may also be enhanced.

\section{LIMITATIONS OF THE STUDY}

It was aimed to establish a research protocol that did not compromise the demand for academic integrity whilst demonstrating direct relevance within the existing inf rastructure of an Indian leprosy hospital. An implication is that the generalizability of the study should be such that similar leprosy hospitals would be able to relate to it. However, the investigators accept that the elevation of ethical issues and external validity as priorities contributed to the difficulty of controlling potentially confounding variables.

It is accepted that the significance of the findings demonstrated in this investigation may be an indication of professional expertise and could therefore be limited in their application among the wider population. The training of leprosy workers and the assessment of their ability to prescribe and manufacture devices may demonstrate the wider potential benefit of this form of intervention.

\section{SOCIOECONOMIC IMPLICATIONS OF THE STUDY}

It has been demonstrated that expensive footwear or footwear meeting with low patient acceptability is not a prerequisite to successful treatment. This is a fundamental implication of this study where the population under review is from a low socioeconomic group and resources are limited. Furthermore, subjects were all ambulant, they were not expected to curtail any normal activity and were not further stigmatized as all the devices were discrete. The significance of this was that no loss of earnings was experienced during the period under review and no alterations to lif estyle were imposed. It is suggested that for these reasons compliance was readily embraced. Enthusiasm for the intervention was apparent and may have been a factor contributing to the generally successful outcome.

\section{Conclusion}

The apparent efficacy of podiatric orthoses as an adjunct to the treatment of ulceration may be greeted with restrained enthusiasm. Unless the treatment can be maintained and developed within the social and cultural parameters affecting leprosy sufferers and workers, the effectiveness of podiatric orthotic intervention remains speculative.

This project shall continue to assess the long-term effects of orthoses as a treatment and as a prophylactic measure in the prevention of recurrence of ulceration. By adopting a broader sociomedical perspective it may be determined whether this approach is an acceptable and realistic management option. 


\section{Acknowledgments}

The authors gratefully acknowledge the encouragement of Dr J. Mehta (Honorary President, Poona District Leprosy Committee) whose support generated and continues to sustain the project.

The authors also acknowledge the assistance of Mr P. Shenton and Mr G. Rendall (Department of Podiatry and Radiography, Queen Margaret College, Edinburgh), Dr E. Alder and Mr G. Bevan (Department of Management and Social Science, Queen Margaret College, Edinburgh).

This study is dedicated to the subjects of the investigation whose enthusiastic cooperation has been invaluable.

Funding for this project has been provided by LEPRA and Queen Margaret College, Edinburgh.

\section{References}

${ }^{1}$ Bauman JH, Girling JP, Brand PW. Plantar pressures and trophic ulceration. J Bone Joint Surgery, 1963; 45(B): 652-73.

2 Schaffe PS, Cavanagh PR. Shoes for the insensitive foot: the effect of a rocker bottom shoe modification on plantar pressure distribution. Foot and Ankle, 1990; 11(3): 129-40.

3 Novick A, Stone J, Birke J, Brasseaux D, Broussard J, Hoard A, Hawkins E. Reduction of plantar pressure with the rigid relief orthosis. J Am Podiatric Med Ass, 1993; 83(3): 115-22.

${ }^{4}$ Rose NE, Feiwell LA, Cracchiolo A. A method for measuring foot pressures using a high resolution computerised insole sensor: the effect of heel wedges on plantar pressure distribution and centre of force. Foot and Ankle, 1992; 13(5): 263-70.

5 Veves A, Masson EA, Fernando DJ, Boulton AJ. Use of experimental padded hosiery to reduce abnormal foot pressures in diabetic neuropathy. Diabetes Care, 1989; 12(9): 653-5.

${ }^{6}$ Holmes GB, Timmerman L. A quantitative assessment of the effect of metatarsal pads on plantar pressures. Foot and Ankle, 1990; 11(3): 141-5.

7 Pollard JP, LeQuesne LP, Tappin JW. Forces under the foot. J Biomed Eng, 1983; 5: 37-40.

${ }^{8}$ McLaughlin P. A Comparative Study into Two Treatments for Metatarsalgia Using In-Shoe Plantar Pressure Measurement System. 1993 BSc. Hons Health Studies Dissertation, Queen Margaret College.

9 Kulkarni VN, Antia NH, Mehta JA. Newer designs in foot-wear for leprosy patients. Ind Lepr, 1990; 62(4): 483-7.

${ }^{10}$ Coleman WC. Shoe gear for the insensitive foot. Clin Podiatric Med Surg, 1987; 4(2): 459-70.

11 Patil KM, Babu TS, Oomen PK, Srinivasan H. Foot pressure measurement in leprosy and footwear design. Ind J Lepr, 1986; 58(3): 357-66.

12 Price EW. Studies on plantar ulceration in leprosy. VI. The management of plantar ulcers. Le pr Rev, 1960; 31(3): 159-71.

13 Ross WF. Etiology and treatment of plantar ulcers. Lepr Rev, 1960; 31: 25-40.

14 Ross WF. Footwear and the prevention of ulcers in leprosy. Lepr Rev, 1962; 33: 202.

15 Ward D. Footwear in leprosy. Lepr Rev, 1962; 34, 94-105.

16 Brand P. Insensitive Feet, A Practical Handbook on Foot Problems In Leprosy. 1981, The Leprosy Mission London.

17 Pring DJ, Casiebanca N. Simple plantar ulcers treated by below knee plaster and moulded double rocker plaster shoe-a comparative study. Lepr Rev, 1982; 53: 261-4.

18 Birke JA, Novick A, Patout CA, Coleman WC. Healing rates of plantar ulcers in leprosy and diabetes. Lepr Rev, 1992; 63(4): 365-74.

19 Brand P. The Insensitive Foot (including leprosy), in: Jahhs MH. (ed.) Disorders of the Foot and Ankle ed 2 Philadelphia, WB Saunders Company 1991.

20 Bickerstaff ER, Spillane JA. Neurological Examination in Clinical Practice. Delhi, Oxf ord University Press, 1989.

21 Watson JM. Preventing Disability in Leprosy Patients. 1986, London, The Leprosy Mission International.

22 Wall B. The clinical assessment of ulcers. The Chiropodist, 1979; Nov., 363-7.

${ }^{23}$ Kundin JI. A new way to size up a wound. Am J Nursing, 1989; (Feb.) 206-207. 
24 Johnson A. Wound assessment. Wound Management, 1993; 4(1): 27-30.

25 Walton RT, Fritschi EP, Umapathy VA. Treatment of plantar ulcers in leprosy patients in the community with adhesive zinc tape. Lepr Rev, 1986; 57: 53-6.

${ }^{26}$ Price H. Connective tissue in wound healing, in: Kloth LC, McCulloch JM, Feedar JA. (eds.) Wound Healing: Alternatives in Management. London, F. A. Davis Company 1990.

27 Johnson A. Towards rapid tissue healing. Nursing Times, 1984; (Nov.) 39-43. 